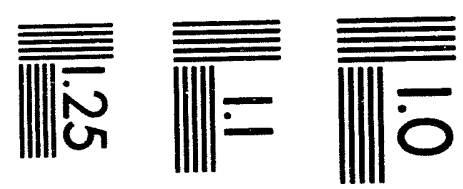

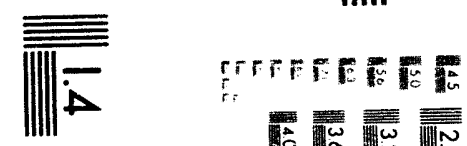

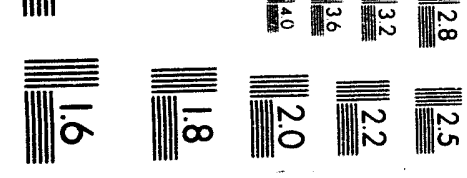



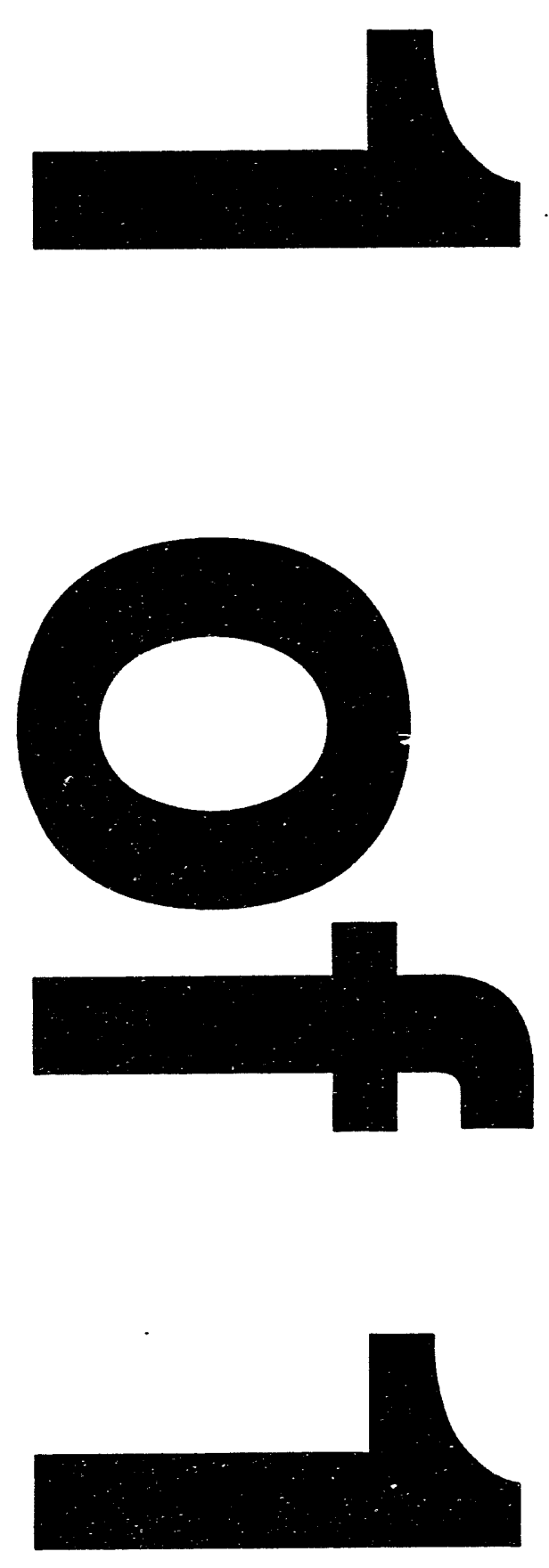


\section{SLOW COLLISIONS OF MULTICHARGED IONS WITH METAL SURFACES}

F.W. Meyer

Oak Ridge National Laboratory, Oak Ridge, Tennessee USA 37831-6362

ABSTRACT

Some recent experimental results in the area of multicharged ion-surface interactions are summarized. Discussed are measurements of projectile K-Auger electron emission during interactions of hydrogen-like multicharged ions with clean and cesiated metal surfaces, measurements of total electron yields for various multicharged ions incident on metal targets, and measurements of projectile angular scattering during grazing metal surface collisions. The various experimental results are presented to illustrate progress in the understanding of multicharged ion-surface interactions in the area of above-vs. sub-surface neutralization and relaxation processes, as well as to identify certain aspects of such interactions where the picture is as yet still incomplete.

\section{INTRODUCTION}

In the past few years, the neutralization and relaxation of slow multicharged ions during interactions with surfaces has been the focus of numerous studies. A variety of experimental approaches have been used, ranging from total electron yield ' and electron spectroscopic ${ }^{2 \cdot 6}$ measurements, to analysis of scattered ions, ${ }^{7}$ and low and high resolution $X$-ray spectroscopy. ${ }^{8-10}$ From these studies, a consistent picture is beginning to emerge of the overall scenario by which the neutralization and relaxation is thought to occur.

It is by now generally accepted that neutralization of multicharged ions can already start well above the surface by classical overbarrier transitions of target valence band electrons to high lying Rydberg levels of the approaching projectile. The critical distance above the surface at which such transitions first become possible is given (in atomic units) by ${ }^{11}$

$$
R_{c}(W, q)=\frac{1}{2 W} \sqrt{8 q+2}
$$

where $W$ is the surface workfunction of the target material and $q$ is the initial charge state of the incident projectile. The principal quantum number, $n_{c}$, into which the valence electrons are initially captured scales ${ }^{11}$ as $W^{-1 / 2}$. For a specific system such as $\mathrm{N}^{6+}$ ions incident on clean $A u, R_{c}$ and $n_{c}$ are approximately 20 a.u. and 7 , respectively. Once electrons have been captured by such resonance neutralization (RN) processes into Rydberg levels of the projectile, the resulting "hollow" atom can relax by a number of different mechanisms. The first is the socalled autoionization (Al) cascade. In each step of this cascade one of the electrons is ejected either into the low energy continuum or into the conduction band $^{11}$ of the metal target (Al' of Fig. 1), while the other de-excites into a level of lower principal quantum number. Such stepwise de-excitation can in principle continue as long as RN persists to balance the resulting projectile electron loss

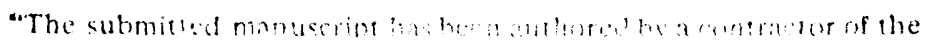

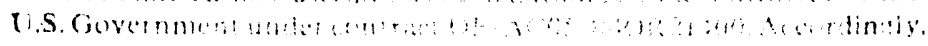

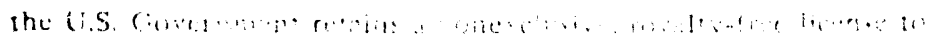

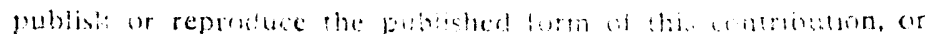
alluw whers to cio so, for U.S. Covernment purpuses."
} 


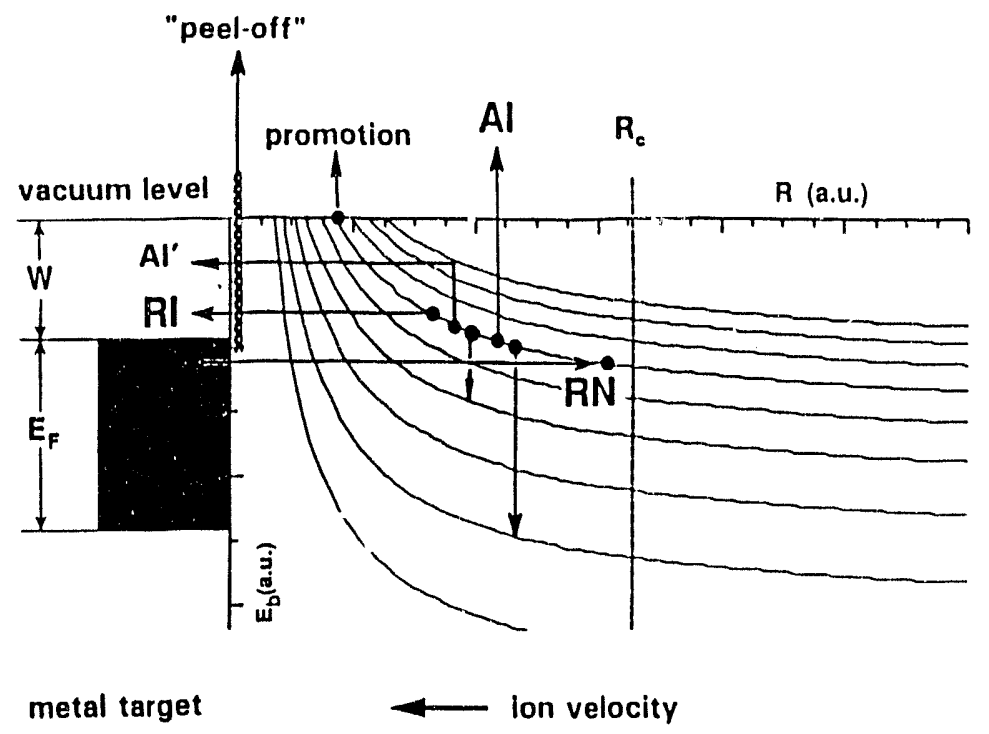

Fig. 1. Possible mechanisms of projectile neutralization, ionization, and electron emission active prior to penetration by the multicharged ion of the metal target surface. (Adapted from Ref. 16.)

Those electrons captured by the projectile into high lying Rydberg levels that have not yet de-excited, may be resonantly ionized (RI) into the metal conduction band or promoted to the continuum as the projectile nears the surface, due to screening by more tightly bound electrons or due to interactions with the projectile image charge, resulting in further above-surface electron emission by the projectile. At point of impact and penetration of the surface, any remaining Rydberg electrons will be "peeled" from the projectile as a result of the sudden screening of the projectile core charge by the high density of valence electrons encountered at the target surface, resulting in further continuum electron ejection or electron injection into the metal target conduction band. The above processes are illustrated schematically in Fig. 1.

Due to the long time scales required for at least two electrons to cascade down to the projectile $\mathrm{L}$-shell in the case of $\mathrm{H}$-like incident projectiles, contributions of above-surface KLL-Auger transitions to the total observed projectile K-Auger electron emission are seen only at very low incident perpendicular velocities. Projectile image-force-acceleration imposes a limit on the lowest perpendicular velocity achievable, with the consequence that the observed above-surface $K$ Auger yield is limited to less than about $20 \%$ of the total K-Auger emission for clean $\mathrm{Cu}, \mathrm{Au}$, or $\mathrm{Ni}$ surfaces. ${ }^{12,4,6}$ After penetration of the surface, screening effects facilitate direct capture into lower projectile $\mathrm{n}$-levels (for $\mathrm{N}^{6+}$ projectiles, $n=2-3$ ), leading to a very fast filling of the surviving $K$-shell vacancies by subsequent $\mathrm{K}$-Auger or $\mathrm{K}_{a}$ radiative decay. The total projectile $\mathrm{K}$-Auger electron emission thus arises predominantly as a result of sub-surface processes. The contribution of sub-surface Auger electron emission to the total electron yield is amplified as a result of the secondary electron cascade processes that the emitted high energy projectile Auger electron can initiate in the target bulk. Indeed, the K- 
Auger electron induced cascade has been found to dominate the potential electron emission yield measured for light $\mathrm{H}$-like ions at impact velocities larger than $10^{7}$ $\mathrm{cm} / \mathrm{s}$.

This article is not intended as a comprehensive review of research in this area. Its intent rather is to show recent progress in understanding of the underlying physical processes by discussion of some selected experimental results of studies on low energy multicharged ion surface interactions obtained at various laboratories. Comparisons of the measurement results and results of modelling studies of above- and sub-surface electron emission are presented and discussed within the framework of the above-outlined scenario.

\section{EXPERIMENTAL APPROACHES}

In order to achieve the very low incident perpendicular velocities required to observe the above potential electron emission processes, a variety of approaches can be used. One possibility is to use beam deceleration techniques employing targets isolated from ground potential that can be floated to potentials arbitrary close to the ion source potential. At Groningen ${ }^{6}$ such an approach is used for electron spectroscopic measurements of projectile Auger electrons emitted during interactions with various clean surfaces. The Vienna group' uses ion deceleration in combination with analysis of the electron emission statistics to determine total electron yields produced by multicharged ion impact on a variety of metal surfaces.

An alternative scheme for obtaining low perpendicular approach velocities is to use undecelerated $\mathrm{keV}$ beams at grazing incidence angles. Such an approach has been used at Caen ${ }^{5}$ and Oak Ridge ${ }^{4}$, and is illustrated schematically in the inset of Fig. 2 , which also shows typical electron spectra acquired for $60 \mathrm{keV} \mathrm{N}^{6+}$ ions incident on clean $A u(100)$ for incidence angles in the range $0.5-19.5^{\circ}$. Varying the perpendicular ion approach velocity by changing the angle of incidence at fixed total ion energy facilitates comparison of above-surface spectral features at different perpendicular velocities, since collisional broadening effects and subsurface neutralization dynamics, both of which strongly affect the subsurface K-Auger peak shape, are strongly energy dependent. One

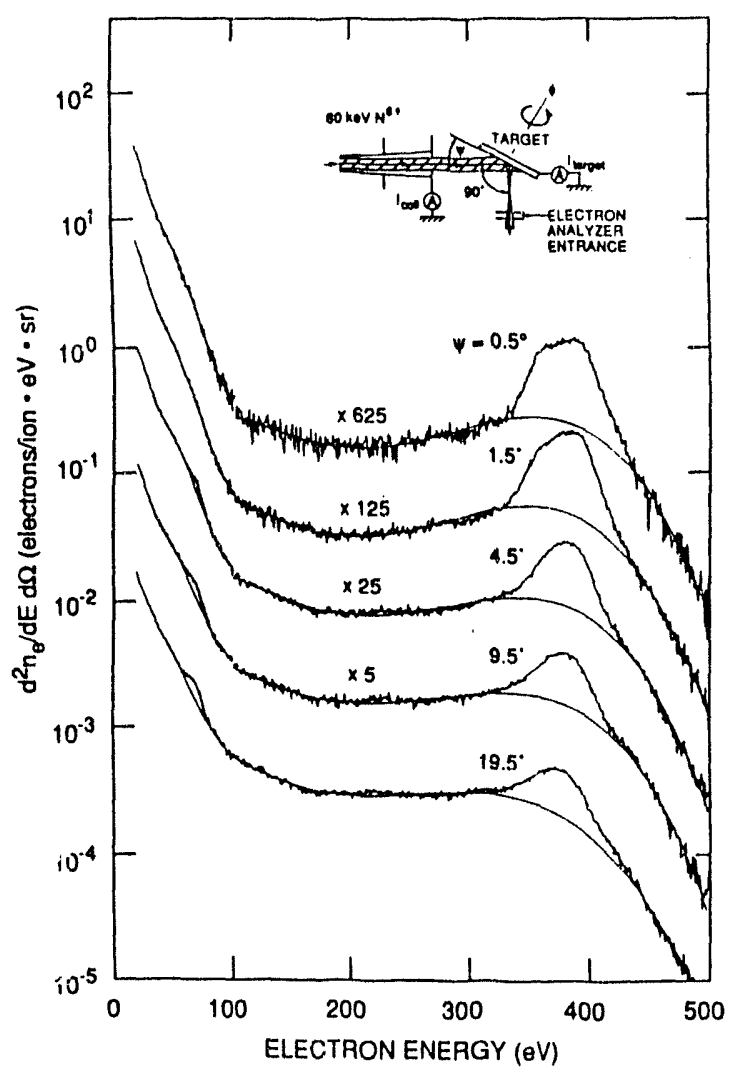

Fig. 2. Electron spectra for $60 \mathrm{keV} \mathrm{N}^{6+}$ incident on $A u(001)$ for various incidence angles (Ref. 4). 
disadvantage of using undecelerated higher energy ion beams for the study of potential electron emission, particularly in the determination of total electron yields, results from the presence of an additional electron emission mechanism, namely kinetic electron emission, whose contribution is not always straightforward to account for.

\section{ABOVE- VS. SUB-SURFACE PROJECTILE K-AUGER EMISSION}

Figure 3a shows normalized K-Auger electron spectra measured at Oak Ridge for incidence angles in the range $0.2-2.4^{\circ}$, after background subtraction using a procedure outlined in detail in Ref. 4 . In this range of incidence angles, a noticeable evolution of the K-Auger peak shape occurs, as a progressively more intense component peaked at about $350 \mathrm{eV}$ is seen to appear in addition to the main sub-

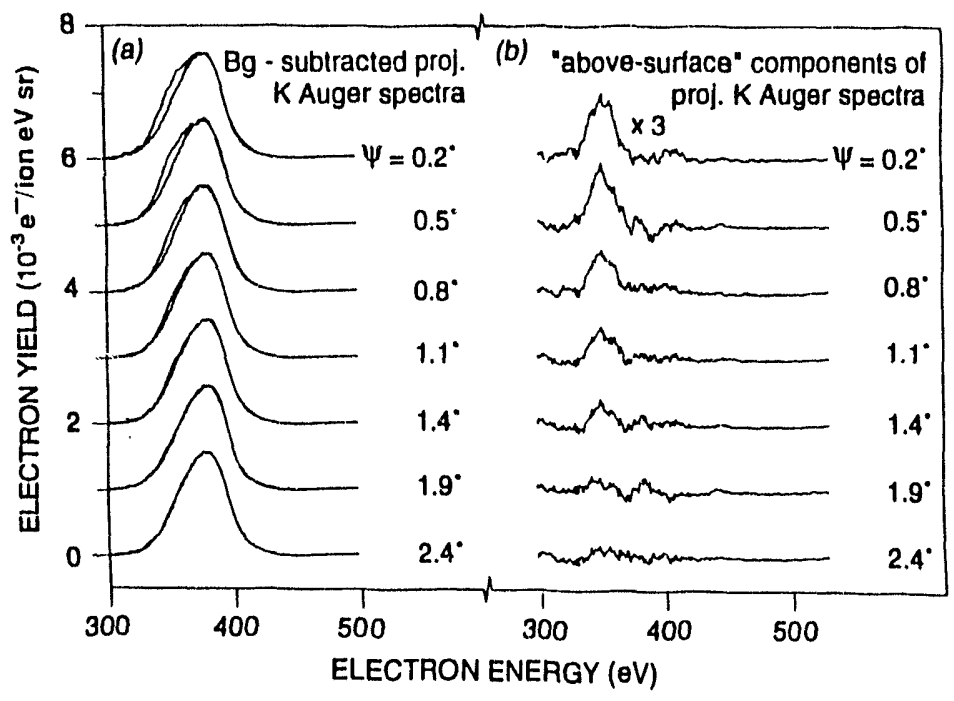

Fig. 3. Background subtracted projectile K-Auger spectra at extreme grazing incidence angles for $60 \mathrm{keV} \mathrm{N}^{6+}$ ions on Cu(011), with superimposed scaled $7.4^{\circ}$ reference spectra (a), and (b) after subtraction of same (Ref. 12).

surface K-Auger lineshape peaked at about $380 \mathrm{eV}$. Prior to the appearance of this additional component, i.e., at incidence angles larger than $2.4^{\circ}$, the shapes of the background-subtracted spectra were found to remain remarkably constarit, the only variation being in the overall intensity. The peak energy of the additional component is consistent with a mininium L-shell population at the time of the $\mathrm{K}$ Auger decay, as is expected from the slow above-surface autoionization cascade. This "above-surface" component is shown in Fig. $2 \mathrm{~b}$ as a difference between a particular grazing angle spectrum and a representative large incidence angle $\left(\Psi=7.4^{\circ}\right)$ spectrum that has been superimposed on it in Fig. 3a, after scaling to have the same intensity at $380 \mathrm{eV}$ (where only sub-surface emission contributes) as the grazing angle spectrum. The above-surface KLL component becomes much more prominent when decelerated ion beams are used due to reduced Doppler broadening effects, as is illustrated in Fig. 4 by a recent electron spectrum 
measured by the Groningen group ${ }^{6}$ for 2 $\mathrm{keV} \mathrm{N}^{6+}$ ions incident at $5^{\circ}$ on clean $\mathrm{Ni}(110)$.

The inverse perpendicular velocity dependence of the intensities of the sub-and above-surface $\mathrm{K}$-Auger components is summarized in Fig. 5, where the integrated $\mathrm{K}$ Auger peak areas are shown for incidence angles up to $20^{\circ}$ (corresponding to the minimum investigated inverse perpendicular velocity of 7 a.u. ${ }^{-1}$ ). The open and solid symbols refer to measurements first published in Refs. 4 and 12, respectively. Similar results have also been obtained ${ }^{4}$ for a Au(011) single crystal target. The identification of the main component of the projectile K-Auger peak as sub-surface emission is supported by the observation of significant target azimuth dependence found for the intensity of this component. The experimentally observed target azimuth dependence as well as the $v_{\perp}^{-1}$ dependence is in excellent accord with a Monte Carlo simulation of the sub-surface K-Auger emission, also shown in Fig. 5. The simulation, described in detail in Refs. 4 and 13, assumes a two step mechanism in the sub-surface K-Auger emission, the first consisting of the filling of the L-shell, described by an adjustable rate $R_{L}$, and the second the KLL transition itself whose rate $R_{k}$ is known. The time evolution of the resulting three component system is solved along actual ion trajectories calculated using

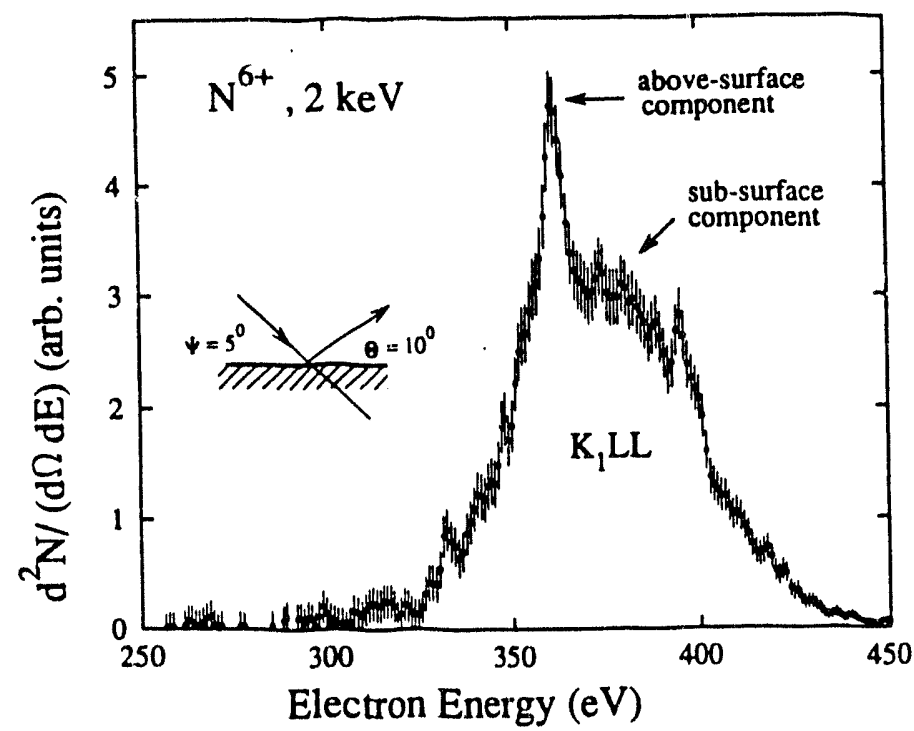

Fig. 4. Electron spectrum measured by Das and Morgenstern for $2 \mathrm{keV} \mathrm{N}^{6+}$ ions incident on Ni(110) at $5^{\circ}$ (from Ref. 6).

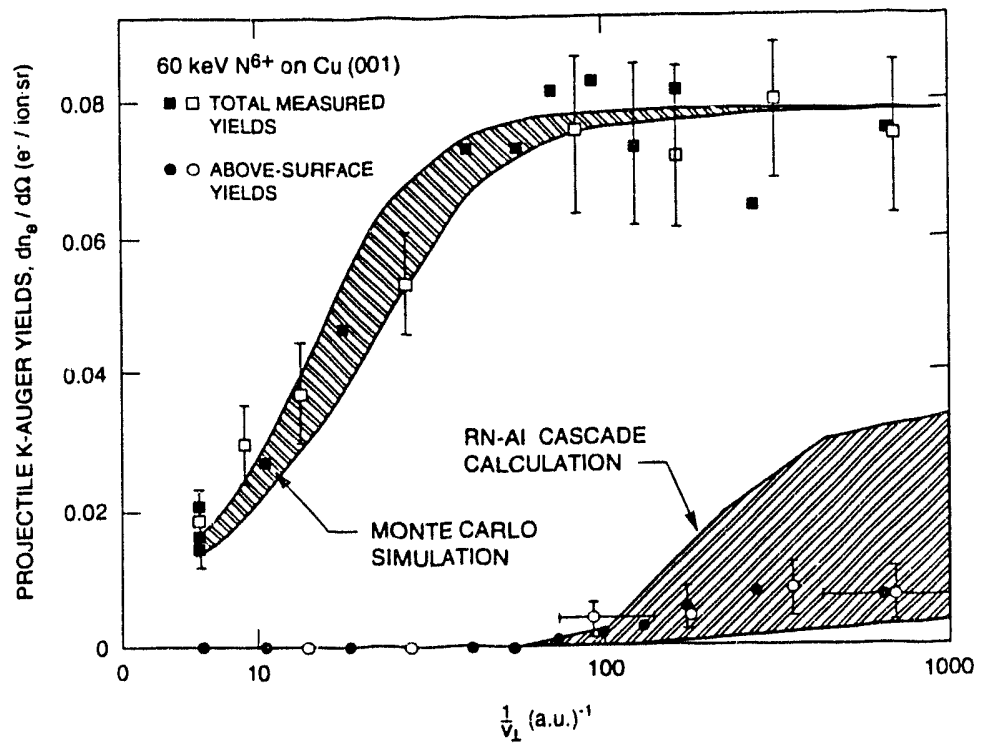

Fig. 5. Above- and sub-surface K-Auger yields (squares and circles, resp.) for $60 \mathrm{keV} \mathrm{N}^{6+}$ incident on $\mathrm{Cu}(011)$ vs. $v_{\perp}{ }^{-1}$, together with simulation results for same (Ref. 12). 
the MARLOWE code. ${ }^{14}$ Transport of the emitted Auger electron to the target surface is treated using tabulated electron inelastic mean free paths. The band delimiting the sub-surface simulation results in Fig. 5 represents the variation in simulated K-Auger yields obtained within the $5^{\circ}$ uncertainty range about the nominal [110] mechanical azimuth setting.

Also shown in Fig. 5 are the above-surface K-Auger peak areas. As can be seen, there is a clear saturation of the above-surface K-Auger electron emission at small perpendicular velocities, as also observed by Das and Morgenstern ${ }^{6}$, which is ascribed to projectile image-potential acceleration. In addition, the results show a threshold of above-surface K-Auger electron emission in the inverse perpendicular velocity interval $57-73$ a.u..$^{-1}$ (incidence angle range $2.4^{\circ}>\psi>1.9^{\circ}$ ), in excellent agreement with classical over-the-barrier simulation ${ }^{11}$ results for the above-surface resonant-neutralization/autoionization (RN-Al) cascade, also shown in Fig. 5. This calculation features a self-consistent and dynamical treatment of the resonance neutralization process and the projectile effective charge, that allows for multiple electron capture as well as loss. The calculation explicitly includes the effect of the dynamical image potential both on the projectile energy levels as well as on the projectile trajectories. Image-potential-acceleration of the projectile is thus accounted for. The indicated band of results shown in Fig. 5 represents the variation obtained under different assumptions for the forms of the image potentials and screening functions, as well as different choices of the Auger rates (see Ref. 11 for additional details).

\section{FURTHER EVIDENCE OF IMAGE CHARGE ACCELERATION}

The projectile image charge acceleration which causes the above noted saturation of the above surface K-Auger yields has also been directly observed in recent measurements of multicharged ion scattering in grazing surface collisions. As is illustrated schematically in Fig. 6, the attractive image interaction force

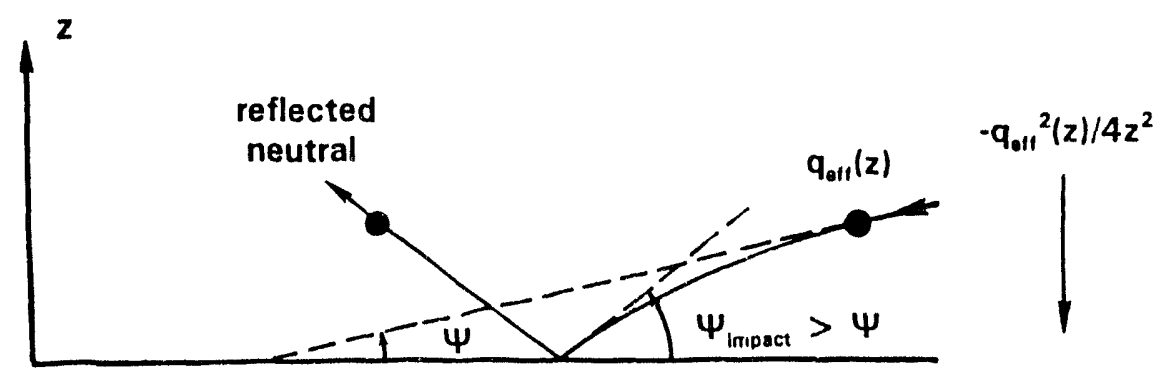

Fig. 6. Effect of image charge acceleration on incident and scattered projectile trajectories.

exerted on the projectile during its approach to the surface increases its incidence angle at impact. In the specular reflection regime, this steeper incidence angle results in a correspondingly larger reflection angle which can be experimentally measured. It is noted that image force effects are largely absent along the receding 
projectile trajectory, since the vast majority of the ions are scattered as neutrals at the investigated energies. The deviation of the observed scattering angle from the asymptotic incidence angle permits experimental determination of the perpendicular energy gained by the projectile as a result of the image charge interaction. The perpendicular energy gain in turn gives direct information on the degree of projectile neutralization occurring above the surface, since it is essentially determined by the evolution of the projectile effective charge as a function of above-surface distance. Figure 7 shows projectile energy gains obtained by Winter ${ }^{7}$ for $25 \mathrm{keV} \mathrm{Xe}^{a+}$ ions incident on $\mathrm{Fe}(110)$ at $0.8^{\circ}$. The dashed line in the figure is the calculated energy gain obtained under the assumption of step-wise neutralization of the projectile (i.e., step-wise decrease of projectile charge) at critical distances given by Eq. (1), and shows remarkable agreement with the data, given the simplicity of the model. Even better agreement with the data is obtained using the simulation of Ref. 11 , which, as mentioned in the previous section, is based on a dynamic and selfconsistent treatment of above-surface neutralization and relaxation. The additional energy gain obtained for the higher charge states using the latter simulation arises mainly as a result of transient existence of the projectile as a negative ion in the final stages of the above-surface neutralization process, which is not accounted for in the simple static "stair-case" model. It is noted that the energy gains inferred from the measurements of Winter are entirely consistent with the perpendicular velocities at which saturation is observed in the above-surface K-Auger yield results.

Evidence for projectile image charge acceleration has also been found in total electron yield measurements by the Vienna group. Using decelerated ions in combination with their electron emission statistics analysis technique, they found a saturation in their electron yield results below critical perpendicular projectile approach velocities that were charge dependent, very reminiscent of the saturation in the above-surface K-Auger yields described above. From an analysis of these critical approach velocities they were able to infer projectile energy gains ${ }^{1}$ for incident projectile charge states up to +79 . Their results are shown in Fig. 8. From the excellent agreement between their experimental results and the staircase model result shown as the dashed line in the figure, it would appear that this simple model has validity even for the very high charge states investigated in this experiment. 


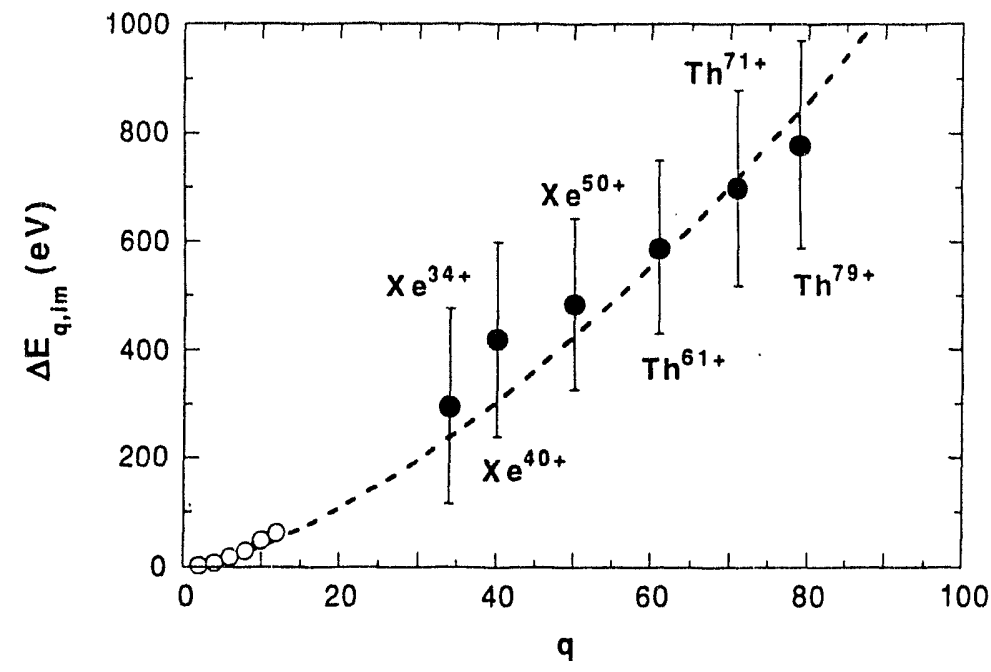

Fig. 8. Energy gains due to projectile image charge acceleration inferred by Aumayr et al. from low velocity saturation of total electron yields (from Ref 1 ).

\section{CESIATED AU(110) SURFACE MEASUREMENTS}

To determine whether the effects of image acceleration could be reduced by the use of low work function surfaces for which, according to Eq. (1), neutralization should start already at much larger above-surface distances, measurements of projectile K-Auger electron emission were performed at Oak Ridge using a cesiated Au surface ${ }^{15}$. Typical results are illustrated in Fig. 9. For the $1.4^{\circ}$ and $0.7^{\circ}$ incidence measurements (top two panels of Fig. 9), the surface was first prepared by $\mathrm{Cs}$ dosing and the change in work function (relative to that for clean Aul measured. In situ thermal desorption was then employed to remove the Cs overlayer, after which another electron spectrum was acquired (right half of each panel). Subsequent to this measurement the loss of Cs was confirmed by a second work function determination. The only difference in experimental conditions between the two spectra was thus the amount of Cs coverage. While significantly reducing the $\mathrm{Cs}$ coverage in each case (see measured work function changes noted in each panel), the thermal desorption cycles were unsuccessful in completely removing the $\mathrm{Cs}$ overlayer. For this reason, the spectra for the smallest incidence angle were measured in reverse order, starting with a freshly sputter cleaned sample.

As has been previously verified both for $\mathrm{Au}$ and $\mathrm{Cu}$ targets, ${ }^{4,12}$ at an incidence angle of $5^{\circ}$ the K-Auger peak arises solely from sub-surface neutralization and relaxation processes. In the present measurements, the shapes of the $5^{\circ}$ K-Auger peaks, also shown in Fig. 9, were found to be independent of Cs coverage in the $0-0.7$ monolayer range investigated, and were used as estimates of the subsurface components in the more grazing incidence angle spectra in a manner already described in the previous section. As can be seen from the spectra shown on the right in Fig. 9, pronounced above-surface components are evident in all the grazing incidence spectra even in the case of minimal Cs coverage. Furthermore, 
significant enhancements of these above-surface K-Auger components were observed for the reduced work function surfaces produced by $\mathrm{Cs}$ deposition (spectra on left in Fig. 9).

From Eq. (1) it can be seen that, for the work function reductions indicated in Fig. 9, more than a factor of two increase in the above-surface critical distance is realized. Simulation results from the previously described over-thebarrier above-surface neutralization code, modified ${ }^{15}$ to permit treatment of a cesiated surface, suggest that the dominant effect of the work function reduction on the simulated above-surface yields is the suppression of the image acceleration of the ion in front of the cesiated surface at large distances due to the screening by the charge cloud of electrons already captured in outer shells. The increased interaction time available for the Auger cascade resulting from the increased critical distance was found to be largely offset by slower Auger rates in the higher initially populated n-shells.

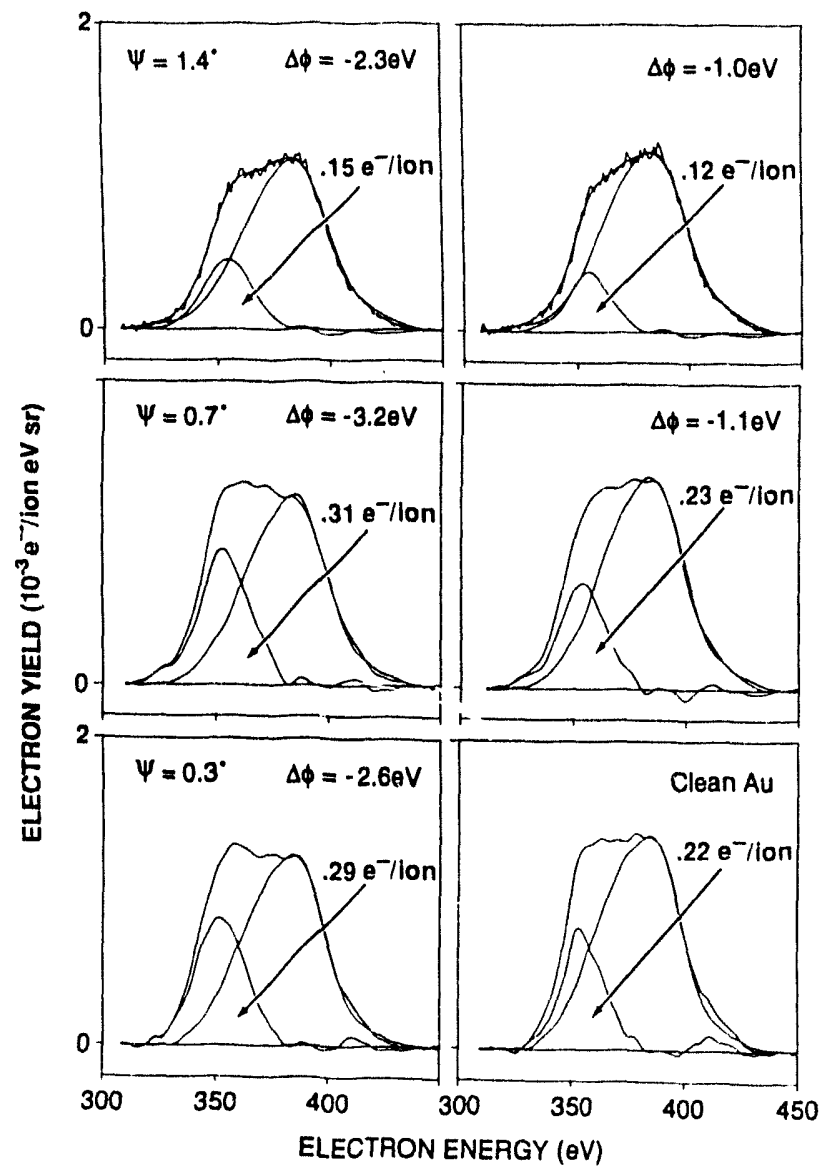

Fig. 9. Background subtracted projectile KAuger peaks for $60 \mathrm{keV} \mathrm{N}^{6+}$ grazingly incident on cesiated and clean $A u(110)$, together with $5^{\circ}$ reference spectra used to estimate the subsurface components. (Ref. 15)

\section{TOTAL ELECTRON YIELD RESULTS}

From the preceding discussion it can be seen that study of projectile K-Auger electron emission during low energy multicharged ion-surface interactions can provide significant information regarding above- and sub-surface neutralization dynamics. Measurements of total electron yields can provide complementary and additional important insights, in that the total yields should manifest more directly the various potential emission processes enumerated in the introduction, including the initial stages of the above-surface autoionization cascade, promotion, and "peel-off." Figure 10 shows the incident velocity dependence of the total electron yield measured' by the Vienna group for $\mathrm{Th}^{71+}$ incident on clean Au. The rise in electron yield with decreasing impact velocity is consistent with the increased electron-emission contribution of the above-surface Al cascade. The already noted 


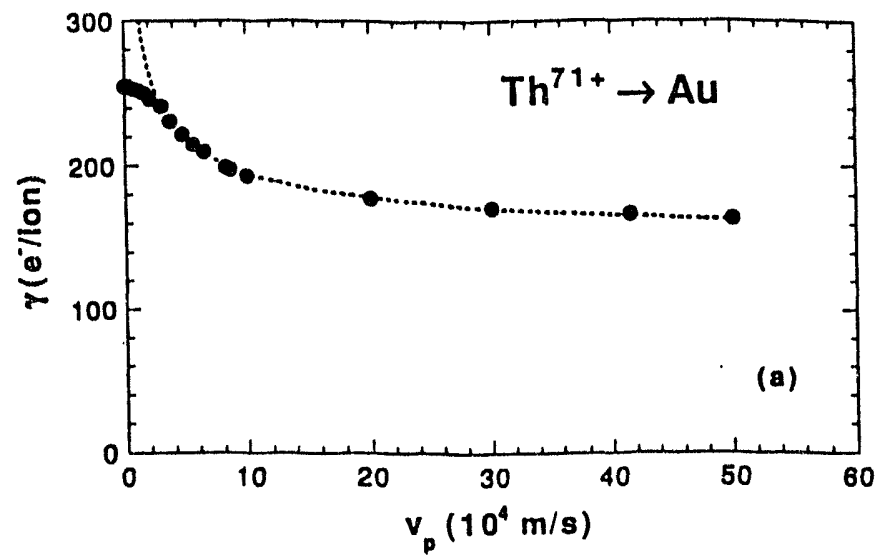

Fig. 10. Total electron yields measured by Aumayr et al. for $\mathrm{Th}^{71+}$ incident on clean $\mathrm{Au}$, as a function of incident projectile velocity (from Ref. 1).

saturation of electron yields is consistent with above-surface projectile image charge acceleration during the course of the stepwise neutralization of the projectile. Model calculations indicate that at the high velocity limit of their measurements, the dominant contribution to the electron yield should be due to electron "peel-off" and promotion, whose combined maximum contribution (which assumes $100 \%$ reflection of the low energy "peel-off" electrons from the surface) is roughly equal to the projectile charge. As can be seen from the figure, the experimental yields at the upper extreme of the measured velocity range, on the other hand, are at least a factor of two higher.

Also puzzling is the observation of projectile core effects in their so-called $\gamma_{\infty}$, the velocity independent components of the measured potential electron yields, which snould consist primarily of "peel-off" and promotion electrons. The processes giving rise to such electrons are expected to be largely independent on projectile core, depending rather mainly on the initial projectile charge state. Table I shows some examples of core effects found by Kurz et al. ${ }^{16}$

Table I: $y_{\infty}$ values for $6+$ and $10+$ projectiles incident on clean polycrystalline Au (from Ref. 16).

$\begin{array}{ll}\frac{\underline{L}_{\infty}}{\mathrm{Ne}^{10+}} & 13 \text { éctile } \\ \mathrm{Ar}^{10+} & 7.8 \\ \mathrm{~N}^{6+} & 5.0 \\ \mathrm{Ne}^{6+} & 3.7 \\ \mathrm{Ar}^{6+} & 3.0\end{array}$

A similar trend was seen in total electron yield measurements ${ }^{17}$ at Oak Ridge for $\mathrm{N}^{\mathrm{a}}$ ions incident on clean $\mathrm{Au}(011)$ at $20^{\circ}$ in the perpendicular velocity range $.09-.19$ a.u., which studied the target azimuth orientation dependence of the electron emission. Typical measurement results are shown in Fig. 11. The striking azimuthal variations in the electron yields seen in the figure are ascribed to kinetic 
electron emission, on the basis of calculations of projectile energy loss using MARLOWE (dashed line in figure), while the target azimuth independent yield differences (e.g., $\mathrm{N}^{6+}-\mathrm{N}^{5+}$ ) is attributed to potential emission.

Simulation
results 17 for abovesurface electron emission processes suggest that, in the investigated velocity regime, the above-surface Auger cascade and promotion to the continuum make only minor contributions to the electron yield. In addition, the maximum possible contribution from peel-off electrons to the yield difference between $\mathrm{N}^{5+}$ and $\mathrm{N}^{6+}$,

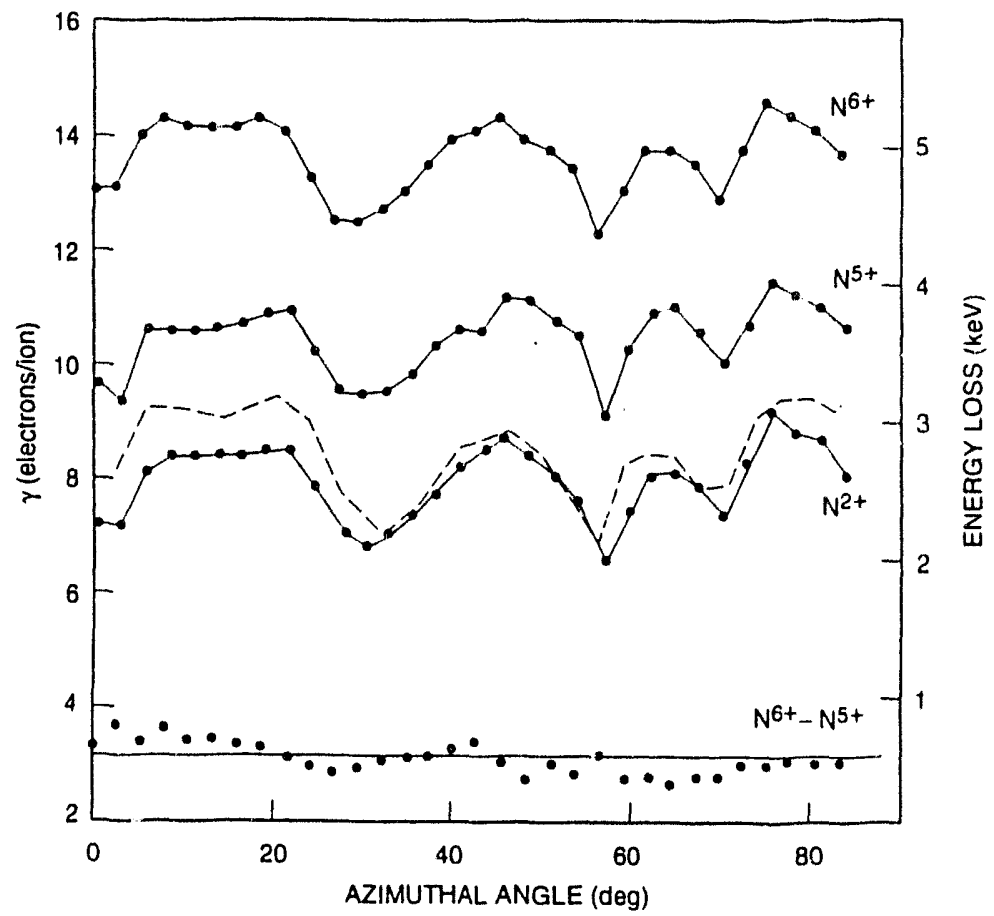

Fig. 11. Target aximuth dependence of the total electron yields for $30 \mathrm{keV} \mathrm{N}^{\mathrm{q}}$ ions incident on Au(011) - solid lines; dashed line - MARLOWE results for projectile energy loss (Ref. 17). also shown in Fig. 11, is on the order of one electron. The observed yield difference of 3.5 e/ion can thus not be accounted for by above-surface processes alone. It was concluded that a major contribution to the observed yield difference must be due to the presence of the initial $\mathrm{K}$-shell vacancy on the incident $\mathrm{N}^{6+}$ ion. The sub-surface filling of this inner shell vacancy in the first $30 \AA$ of the target gives rise to isotropically emitted $380 \mathrm{eV}$ electrons, which can in turn create low energy secondary electrons. Using published data ${ }^{18}$ of electron induced secondary electron emission, a rough estimate of $1.5-2 \mathrm{e} /$ ion is obtained. "Indirect" potential electron emission ${ }^{17}$ due to secondary cascade processes within the target bulk is thus believed to be an important contributor to potential emission for the above system. It is conceivable that similar sub-surface potential emission processes may also make significant contributions to the electron yields observed for projectiles of much higher charge state.

\section{ACKNOWLEDGEMENTS}

The work from Oak Ridge described in this paper was supported by the Office of Basic Energy Sciences, Division of Chemical Sciences, and the Office of Fusion Energy, Division of Applied Plasma Technology, of the U.S. Department of Energy, under Contract No. DE-AC05-840R21400 with Martin Marietta Energy Systems, Inc. 


\section{REFERENCES}

${ }^{1} F$. Aumayr, H. Kurz, D. Schneider, M.A. Briere, J.W. McDonald, C.E. Cunningham, and HP. Winter, Phys. Rev. Lett. 71, 1943 (1993); H. Kurz, K. Töglhofer, HP. Winter, F. Aumayr, and R. Mann, Phys. Rev. Lett. 69, 1140 (1992).

${ }^{2}$ L. Folkerts and R. Morgenstern, Europhys. Lett. 13, 377 (1990).

${ }^{3}$ H.J. Andrä, A. Simionovici, T. Lamy, A. Breriac, G. Lamboley, J.J. Bonet, A. Fleury, M. Bonnefoy, M. Chassevent, S. Andriamonje, and A. Pesnelle, Z. Phys. D 21, S135 (1991). ${ }^{4}$ F.W. Meyer, S.H. Overbury, C.C. Havener, P.A. Zeijlmans van Emmichoven, and D.M. Zehner, Phys. Rev. A 44, 7214 (1991); F.W. Meyer, S.H. Overbury, C.C. Havener, P.A. Zeijlmans van Emmichoven, and D.M. Zehner, Phys. Rev. Lett. 67, 723 (1991).

${ }^{5} R$. Köhrbrück, N. Stolterfoht, S. Schippers, S. Hustedt, W. Heiland, J. Bleck-Neuhaus, D. Lecler, and J. Kemmler, Phys. Rev. A 48, 3731 (1993); S. Schippers, S. Hustedt, W. Heiland, R. Köhrbrück, J. Bleck-Neuhaus, J. Kemmler, D. Lecler, and N. Stolterfoht, Phys. Rev. A 46, 4003 (1992).

${ }^{6} \mathrm{~J}$. Das and K. Morgenstern, Phys. Rev. A 47, R758 (1993).

${ }^{7} \mathrm{H}$. Winter, Europhys. Lett. 18, 207 (1992); H. Winter, p. 583 in Proceedings, Vlth Intl. Conf. Phys. Highly Charged lons, Manhattan, Kansas, 1992 (AIP Conference Proceedings 274, 1993); H. Winter, C. Auth, R. Schuch, and E. Beebe, Phys. Rev. Lett. 71, 1939 (1993).

${ }^{8}$ M. Schulz, C. Cocke, S. Hagmann, M. Stöckli, and H. Schmidt-Böcking, Phys. Rev. A 44, 1653 (1991).

'J.J. Bonnet, A. Fleury, M. Bonnefoy, M. Chassevent, T. Lamy, A. Brenac, A. Simionovici, and H.J. Andrä, Z. Phys. D Suppl. 21, 5343 (1991).

${ }^{10}$ B. d'Etat, J.P. Briand, G. Ban, L. de Billy, J.P. Desclaux, and P. Briand, Phys. Rev. A 48, 1098 (1993).

${ }^{11} \mathrm{~J}$. Burgdörfer, P. Lerner, and F.W. Meyer, Phys. Rev. A 44, 5674 (1991); J. Burgdörfer and F.W. Meyer. Phys. Rev. A 47, R20 (1993).

${ }^{12}$ F.W. Meyer, C.C. Havener, and P.A. Zeijlmans van Emmichoven, Phys. Rev. A 48, 4476 (1993).

${ }^{13}$ S.H. Overbury, F.W. Meyer, and M.T. Robinson, Nucl. Instrum. Methods Phys. Res. B 67, 126 (1992).

${ }^{14}$ M.T. Robinson, Phys. Rev. B27, 5347 (1983); O.S. Oen and M.T. Robinson, Nucl. Instrum. Methods Phys. Res. 132, 647 (1976).

${ }^{15}$ F.W. Meyer, L. Folkerts, I.G. Hughes, S.H. Overbury, D. M. Zehner, J. Burgdörfer, and P. A. Zeijlmans van Emmichoven, Phys. Rev. A 48, 4479 (1993).

${ }^{16} \mathrm{H}$. Kurz, F. Aumayr, C. Lemell, K. Töglhofer, and HP. Winter, Phys. Rev. A 48, 2182 (1993).

${ }^{17}$ I.G. Hughes, J. Burgdörfer, L. Folkerts, C.C. Havener, S.H. Overbury, M.T. Robinson, D.M. Zehner, P.A. Zeijlmans van Emmichoven, and F.W. Meyer, Phys. Rev. Lett. 71, 291 (1993).

${ }^{18}$ S. Thomas and E.B. Pattinson, J. Phys. D 3, 349 (1969).

\section{DISCLAIMER}

This report was prepared as an account of work sponsored by an agency of the United States Government. Neither the United States Gnvernment nor any agency thereof, nor any of their employees, makes any warranty, express or implied, or assumes any legal liability or responsibility for the accuracy, completeness, or usefulness of any information, apparatus, product, or process disclosed, or represents that its use would not infringe privately owned rights. Reference herein to any specific commercial product, process, or service by trade name, trademark, manufacturer, or otherwise does not necessarily constitute or imply its endorsement, recommendation, or favoring by the United States Government or any agency thereof. The views and opinions of authors expressed herein do not necessarily state or reflect those of the United States Government or any agency thereof. 

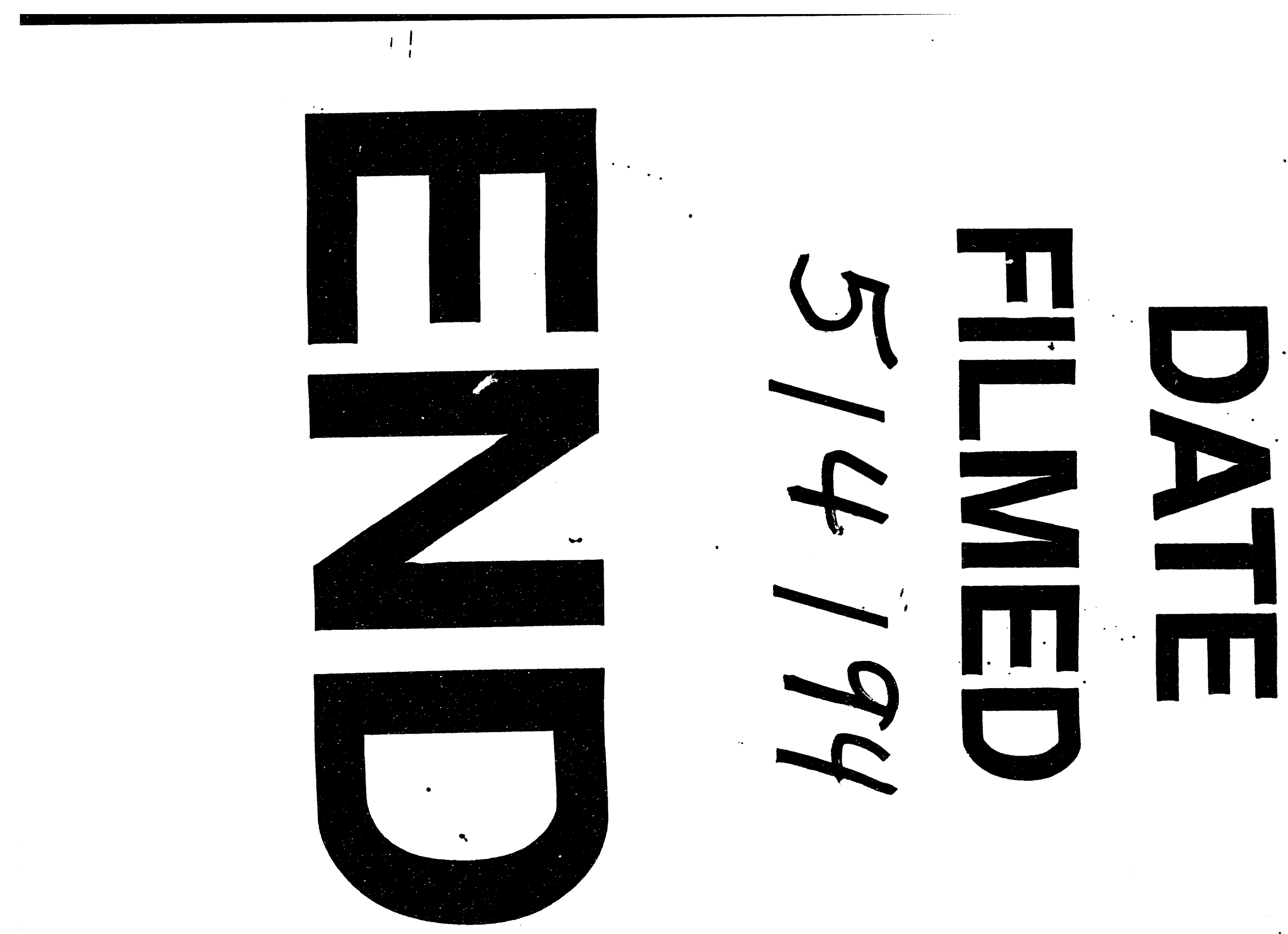
